\title{
The influence of Ca-Mg disorder on the growth of dolomite
}

\author{
BRUNO M. ${ }^{1}$ \\ ${ }^{1}$ Dipartimendo di Scienze della Terra, Università degli Studi \\ di Torino, Via Valperga Caluso 35, Torino, 10125-Italy, \\ marco.bruno@unito.it
}

Dolomite, $\mathrm{CaMg}\left(\mathrm{CO}_{3}\right)_{2}$, is one of the most abundant carbonate mineral in the Earth, but it is still unclear its genesis. In particular, the effect of the $\mathrm{Ca}-\mathrm{Mg}$ disorder on the stability and growth of dolomite is not totally understood.

Recent quantum-mechanical studies [1,2] have shown that the bulk of the dolomite crystal must have an ordered structure to reach the minimum of the energy, whereas the (10.4) surface is more stable when its structure is disordered. By means of these results, a growth model of the (10.4) face has been proposed [2]: the peculiarity of this model consists in the existence of some disordered layers forming at the interface crystal/solution, which arrange in an ordered structure once covered by others disordered layers resulting by the spiral steps propagation.

Starting from these results, we performed empirical calculations on (10.4) slabs of dolomite with increasing thickness and different degree of structural disorder (i.e., different $\mathrm{Ca}-\mathrm{Mg}$ distributions in the layers forming the slab). The calculations were performed by considering the (10.4) slabs of dolomite growing (i) without a crystalline support, to simulate a homogeneous nucleation, and (ii) in epitaxy with the (10.4) face of calcite, to sumulate a heterogeneous nucleation. Interestingly, we observed in both cases (i.e., with and without crystalline support) that disordered (10.4) slabs of dolomite formed by one/two layers are energetically more stable that the ordered (10.4) one. Instead, when the (10.4) slabs of dolomite are thicker (i.e., more than one/two layers), the ordered slab is more stable than the disordered ones. This allowed us to hypothesize a new growth model for dolomite, where the contribution of calcite is not negligible.

[1] Zucchini et al., Calphad 2012, 38, 177; [2] Bruno and Bittarello, Minerals 2018, 8, 323 